\title{
The Study on the Application of European Style in Villa Design \\ Lijuan $\mathrm{Yao}^{1, \mathrm{a}}$ \\ ${ }^{1}$ Hebei Arts Academy, Hebei, 050700 \\ hunter2011@foxmail.com
}

Keywords: European Style, Design, Villa, Style

\begin{abstract}
European-style is one of the most suitable design styles for villa. European style has a long history and has an enormous share of the contemporary villa design. In this paper, we start through the characteristics of European-style in villa design and specific analysis the European-style decorative elements in the design of villa by the two aspects of soft furnishings and hard decorative. And we also discuss the application methods of European-style in villa design in order to play a draw in the villa design activities for designers
\end{abstract}

\section{Introduction}

With the rapid growth of China's economy, the demand for high-end housing industry is also growing, developers gradually began to attach importance to the development of the villa and the villa space design has been referred to the relevant table. European style is undoubtedly one of the most suitable for modern villa style design, in order to build a better home environment, so the advantages of European-style villa perfectly reflected in the design, the paper design in European style villa apply this topic to do simple discussion.

\section{The European-Style Characteristics in Modern Villa Space}

European-style features. Traditional European architecture has the characteristics of large space, its interior space or a large or tall. Thus, European-style decor often fit a larger area of the space, being applied to a large area of the house, in order to better demonstrate its features. European-style layout is generally symmetrical forms and large space each other, in order to reflect the overall momentum of the magnificent grand European architecture. European-style interior biggest feature is the pursuit of luxury, gorgeous visuals and usually emphasize three-dimensional lines, beautifully detailed shape, pattern texture of fine and other means, to create elegant luxury and rich and gorgeous visuals. Meanwhile, European-style is focus on comfort and romantic atmosphere and mellow cultural heritage, under the luxurious and brilliant space yet cozy, elegant and warm atmosphere.

The desirable characteristics of European-style in villa space design. Villa decoration is usually to highlight the level of a person's life and art conservation, highlighting their houses luxury and cultural heritage, European style best embodies these characteristics, but the characteristics of the villa and a large space can also be a good combination of European style. Villa space design should pay attention to the following features

Cultural heritage. In the design of villa space, cultural issues should be taken seriously, a good highlight the occupants of education and cultural taste. European-style long history, have their own unique style features and cultural heritage. European-style villa decorated space, should highlight these Mandarin heritage, such as the marble of the materials used to design the villa space, but also highlights the texture of the entire space, plus walnut furniture decorated with dark brown color is formed, highlights the nostalgic charm of traditional culture temperament [1]. Wallpaper on the walls complemented by classic-style paintings, Roman blinds for the Mandarin tone, horizontal surface display of collectibles and crafts sculptures Mandarin breath better highlight the cultural heritage of the integration space.

Humane. The interior design is the first thing to take into account the characteristics of human nature, for this high-end villa space needs in terms of design, human needs when almost universal, 
and the quality requirements for humane increasingly high. Therefore, in the space of the villa design we should pay attention to added humane factors. Humanized design in modern design hot topic, do pay attention to "people-oriented" the basic principles in the design, requirements designed to try to meet the dual needs of the occupants of physiological and psychological. Villa occupants belong to high-end social groups, they later for psychological needs, so space for modern villa designed to ensure the physical needs of the residents at the same time, but also pay attention to their psychological needs.

The integrity. Under normal circumstances, the architectural features of the villa now has a European element in this respect, it is necessary to adopt European-style villa interior space design, in order to maintain the overall style form both inside and outside the villa coincide with each other. Style overall performance guarantees aesthetic continuity of indoor and outdoor, in line with the occupants and visits by aesthetics, but also in line with the trend of modern living. Therefore, in the space of modern villa designed for indoor and outdoor continuity, but also for soft furnishings and decorative perfect combination of hard, play better advantage of this design, you should consider the use of European-style as a whole.

The ecological. Villa located in a better environment is generally better suburbs, ecological environment, the villa's occupants tend to conduct its own environmental and quality of life have higher requirements, they usually ecological requirements are relatively high, and this demand is that no matter indoors or outdoors are present. Therefore, when the villa space design, it is necessary to highlight the green of the interior space, pay attention to ecological elements, rational use of design in green plants, we should pay attention to indoor and outdoor ecology ecological continuity.

\section{The Application of European-style in Villa Hard-Decorated}

The use of space. In hard decorative design, we should in the original layout of the building, combined with the actual needs, the partition, the combination of space, and thus vary in layout, space separated, combined, we must take into account the comfort level of people's requirements and housing this design will play a role in skeleton [2]. European decorative style using major highlight in the space atmosphere, this atmosphere is not only reflected in the large when the entire space, and are often able to reflect the characteristics of the local large space. In a large sense of space tend to emphasize the dynamic, generally have asymmetry, the internal space are generally irregular shape. For example, the use of the ups and downs of the adjacent line, the length of the corresponding, depth dependent, achieves the purpose of the entire space of relaxation.

Functional partitioning. Segmentation and combination of space, making the interior space formed can have different functions, including dynamic open space and static space, virtual mobility space [2]. To a large space becomes richer in the process, but without losing the elegance and luxury atmosphere, the need for space and their own characteristics have a full understanding and knowledge. Of course, the purpose of the design is to be processed transformation in a limited space, in order to achieve the goal of miraculous. For example, for living and dining rooms (open space) partition, European-style decorative designs often do not need to block entities, the use of color change and reflection principle of separate space and layout to achieve basic space on the partition function, size and shape can be more ambitious space preserved.

The maintenance of space body. Top surface treatment. European-style ceilings often has strong colors, exquisite styling and ornate decorations and to achieve the atmosphere, luxurious decorative effect. The top of the living room usually has a large pool lights, pool lights edge gypsum line with the general pattern of the blank, and then luxury chandelier (Chandelier) to manufacture luxurious atmosphere; bedroom at the top to reduce the complexity of textures and lines, pay attention to reflect elegant and welcoming atmosphere. At the junction with the wall more plaster moldings decorated female, to highlight the three-dimensional sense of space and beauty, will use some of the classical European wooden moldings overcast, it can also bring some Mandarin breath.

Wall processing. On the wall treatment, focusing on European-style interface of the Rules and balance, in addition, also stressed that the stability of shape and enrich the wall, using the appropriate 
proportions, to increase a sense of order; not only to multi-wall wallpaper, also have a real fireplace or fake fireplace modeling, and multi-line framing plaster, wallpaper and moldings inside the outer wallpaper usual style and color vary widely, layering protruding wall; the wall modeling (such as TV backdrop ) mostly stone-based, using appropriate lines and texture changes to make the interface more rich sense of detail and attractive; mostly with waist aisle walls or wood paneling to heighten the effect of luxury, classic European-style multi-use and other dark walnut dado wood as raw material to reflect its mellow, simple European style is the use of white paint to show its elegance. Overall wall color choices are generally considered the reflection of light and impact.

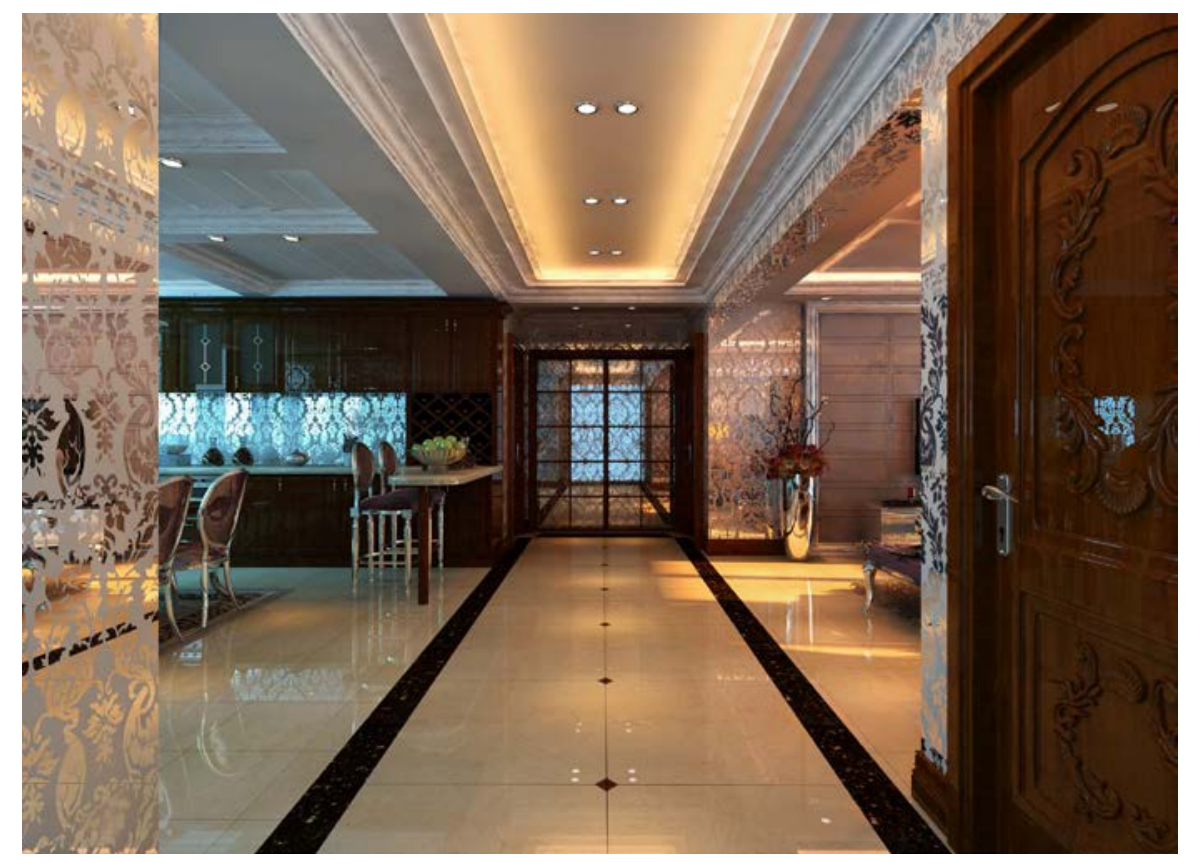

Fig 1. The district features of living and dining

Ground handling. The meaning of ground handling is using some of the processing means on the ground to achieve complementary function of the space division and colors and stripes while retaining the atmosphere of the original space, the Villa interior of the house is a duplex, on the first floor hall floor using stone and it will become rich atmosphere. Marble hall and the whole cast glaze based materials, and at the foot of the wall Stones waveguide line to expand space; also used in the restaurant of the same or similar material, a modified four weeks waveguide line pattern inside the ground and hall complement each other; in the living room and restaurant combine the two to form a transmission line at the aisle by color differences and reflection effects, different from both sides of the aisle in the general use of stone or brick, contrast different colors and parquet floor can enhance the richness, showing a gorgeous sense of the ground (as shown in the aisle floor in Figure. 1).

Doors and windows. The upper part of the doors and windows are usually made of continental arc-shaped, in such a way that the main impact of the visual, you can generate a sense of visual extension, giving the feeling of space even more towering. Surrounding these doors are generally decorative stone or wood as large sets of wire material, sets of lines often with shades of multilayer carved into fine lines or patterns with a feeling of relief, and in some cases, sets of lines show will paint on a white paint, but more reserved wood, stone original color and texture effect. The windows and doors decorated with traditional methods, to reflect the antique cultural heritage can serve the high-end atmospheric style and it is one of the important decorative features typical European styles.

\section{The Application of European-style in Villa Soft Furnishings}

Maintenance and adding of open spaces and decorative body. Curtains. European-style curtains emphasize the linear flow of change, gorgeous colors, often with lace, gold rim modified to highlight the atmosphere of luxury. It is in the form of romanticism, based on brisk slender curve pattern composed of flowers, landscapes and some conceptual images, the use of color and the whole 
room curtains colors, creating a romantic, cozy atmosphere. There are also some plain style curtains, patterns and even simple solid color, a warm and elegant style and these are generally used for the bedroom.

Wallpaper and wainscoting. Wallpaper is customary in European style decorative elements, but also the European space important part. Wallpaper patterns varied rich colors, to reflect the elegance gorgeous space; soft texture wallpaper visual sense has a very soft and comfortable visual effect, you can bring out the warm and cozy space; you can choose a more distinctive decor of the room, such as wallpaper painted wallpaper Bible stories and characters, etc. with a strong culture and content, can enrich the cultural heritage in space, you can also paint some of the residents in line with the interests and hobbies as a decorative pattern. Wainscoting is a unique European-style decorative form and mosaic on the wall of wood can be high or low, it is possible to increase the space of layering and rich, often with lines plank surface geometry, in the sunlight and lights changes rich features.

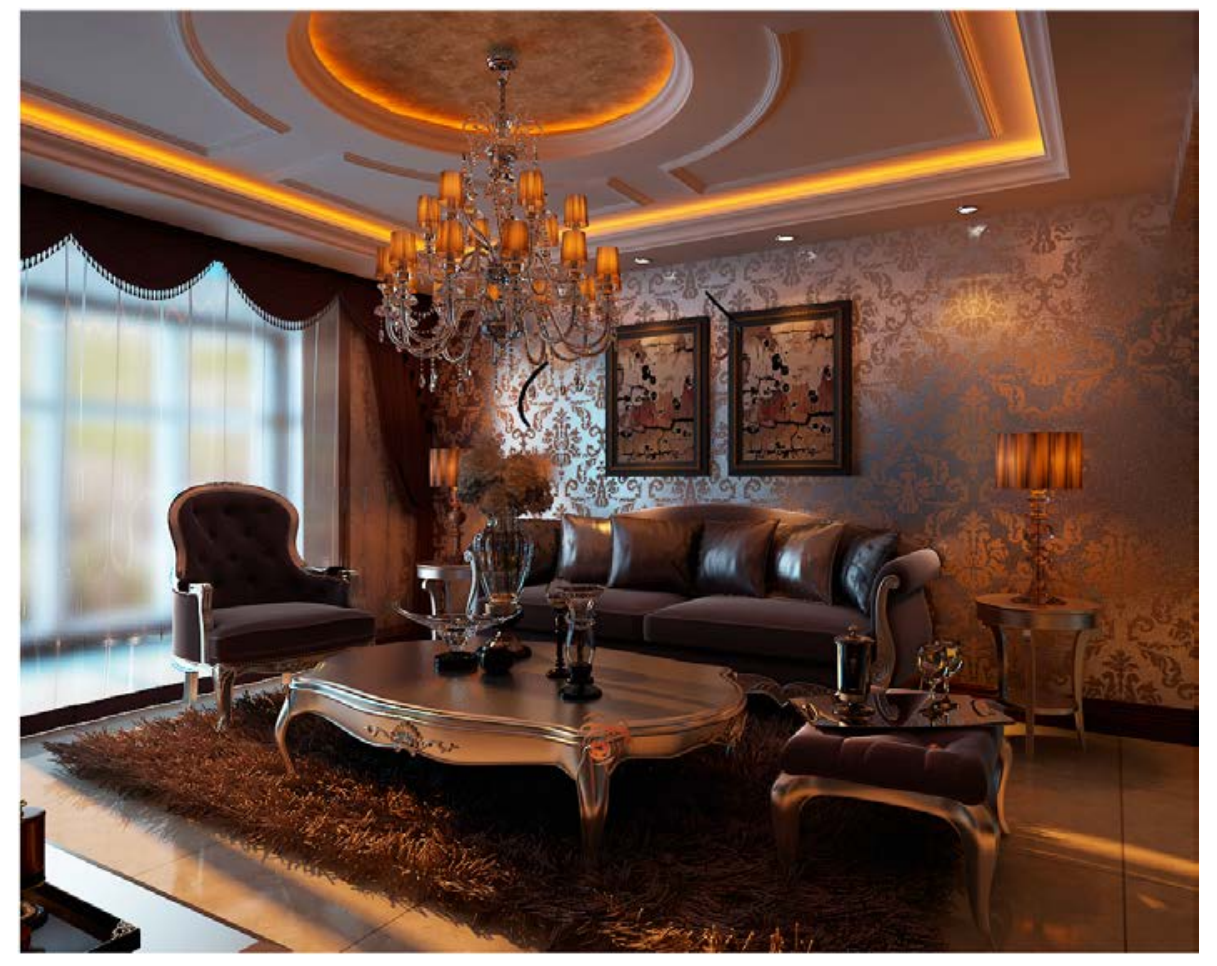

Fig 2. The top surface and wallpaper of the living room

Carpet. European-style carpet bear softening ground space and decorative role, the main role of the ground also by the carpet to play, traditional European carpets pure wool preparation [3]. European carpet texture soft and comfortable, elegant patterns, rich colors and other features colorful, in the living room, a central dining room, bedroom and study can be laid, also can be decorated at the junction of the aisle, and some ribbon. These rugs can make the ground rich layering, enhance occupant comfort, warm atmosphere increase, then the high-end European furniture and objects on display reflect each other in order to heighten lining the entire space atmosphere.

The jewelry and decorative in space. Lighting. Continental Light is synonymous with luxury amenities. From the European classical style of art, imitation ancient European palace style effect. European classical charm lies in its unique historical traces of the years, which reflects the timeless elegance of tolerance represents a host of excellent quality of life. European-style lamp with ornate decoration, strong colors, beautiful shape to achieve elegant, magnificent European palace effect, European-style lights focus on line, shape and color on the carving. Some light will be to deliberately create a vintage effect artificial rust, dark paint, etc., giving the visual classical feel. From the material point of view, European-style lights and more to the resin, copper, forged iron based. Wherein the resin lamp shape a lot, can have a variety of patterns, gold and silver foil affixed seem bright colors, bright color; copper, iron and other shapes is relatively simple, but even more texture. Lighting is to 
create a magnificent European-style interior indispensable element, colored lamps and lighting tangible, is the most effective means to create an indoor atmosphere.

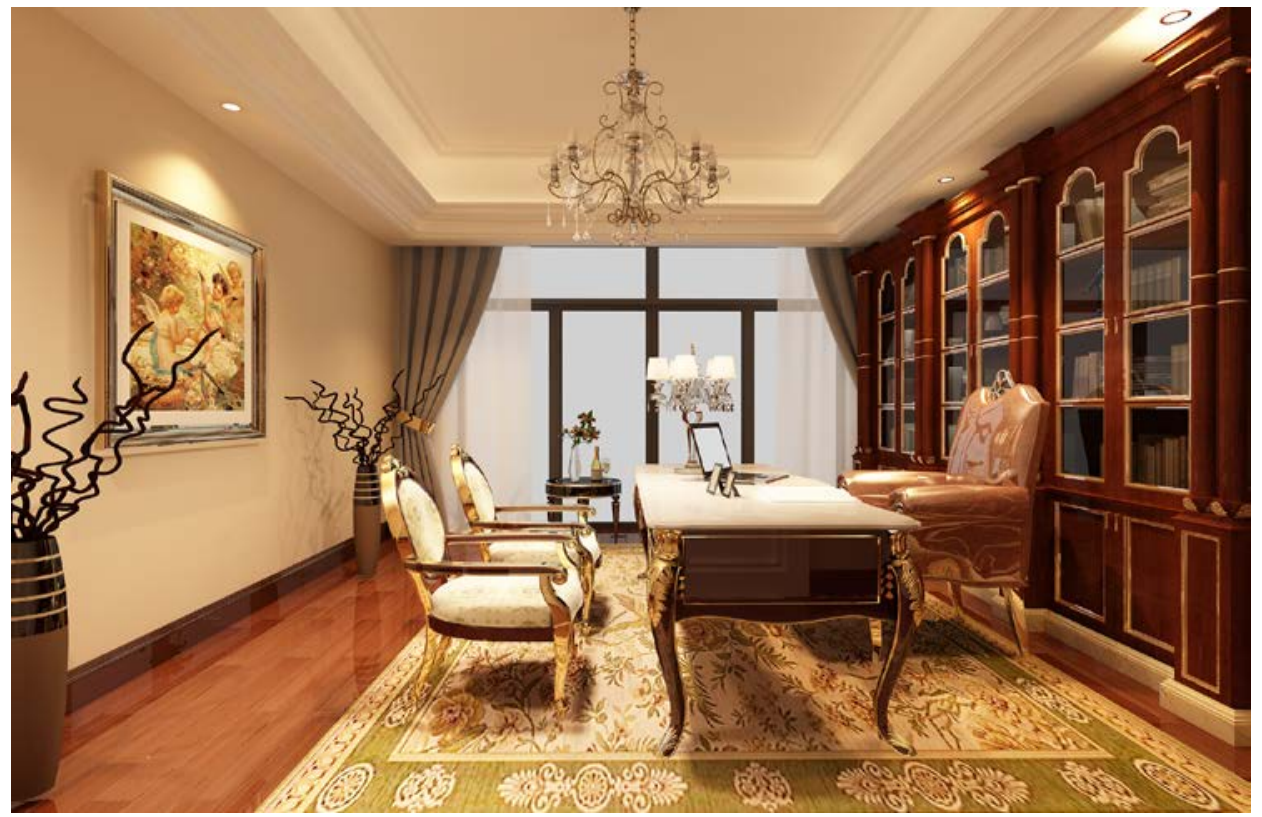

Fig 3. The carpets, furniture and display of study

Display things. Furnishings for interior plays an important role, paintings, mirrors, sculptures and other objects on display at the European-style space everywhere. Decorative mirror display, to reflect the limited space in the visual experience more space, multi-angle vision but also can improve people's experience of visual enjoyment. Paintings and collectibles, space reflect artistic taste, culture, reflecting the cultivation, the quality and status of occupants. Sculptures and watches on display, there are shapes and diverse, rich themes, different attitude, etc., it can be more flexible space rich content, regulation and complementary colors with large pieces of furniture.

Ecological plant. European-style ecological plant to flower-based, European-style floral often symmetrical, balanced pattern appears. Its colorful and rich, atmospheric composition neat, elegant and gorgeous appearance, with elegant, warm yet dignified features. Flower select the above should make these flower arrangements perfect match with the surrounding environment, not only enhance the beauty of space, but also to space into the rich natural flavor, in addition, the flower color should be consistent with the overall color space requirements, and can and outdoor ecological environment a certain connection.

Furniture. Continental furniture exquisite hand-carved fine cut, in part by turning contour and symmetry and rhythmic curve or curved surface, and decorative ornaments gilded copper, leather, etc., is simple in structure, smooth lines and rich colors, strong sense of art, to people's overall feeling is luxurious and elegant, very dignified. The details is carved gilt surface elegant, full of artistic charm. The real European furniture, emphasize the overall decorative effect, complete with a hundred percent pure wood building, with its multi-color solid wood dark red, brown-based embellished pearl white or golden yellow, reflecting the distinguished furniture, and luxurious.

\section{The Integrity Application of European-Style in the Villa Design}

First, in the European style villa interior design, decoration hard reasonable overall space planning division and function, the overall decor reflects the atmosphere of hard luxury style; secondly, matched the curtains, wallpaper, carpet and hard decorative landscaping modifications increase the rich and layered space outside, set the tone for the overall atmosphere; again, all kinds of furniture, display objects, illustration with each other, in the case in line with the tone of space, rich sense of color space, and create a comfortable living environment achieve elegant temperament, into the ecological and cultural heritage; and finally, lighting and colored lights visible color space again 
embellishment, enhanced spatial visual enjoyment, express a romantic, comfortable and elegant atmosphere.

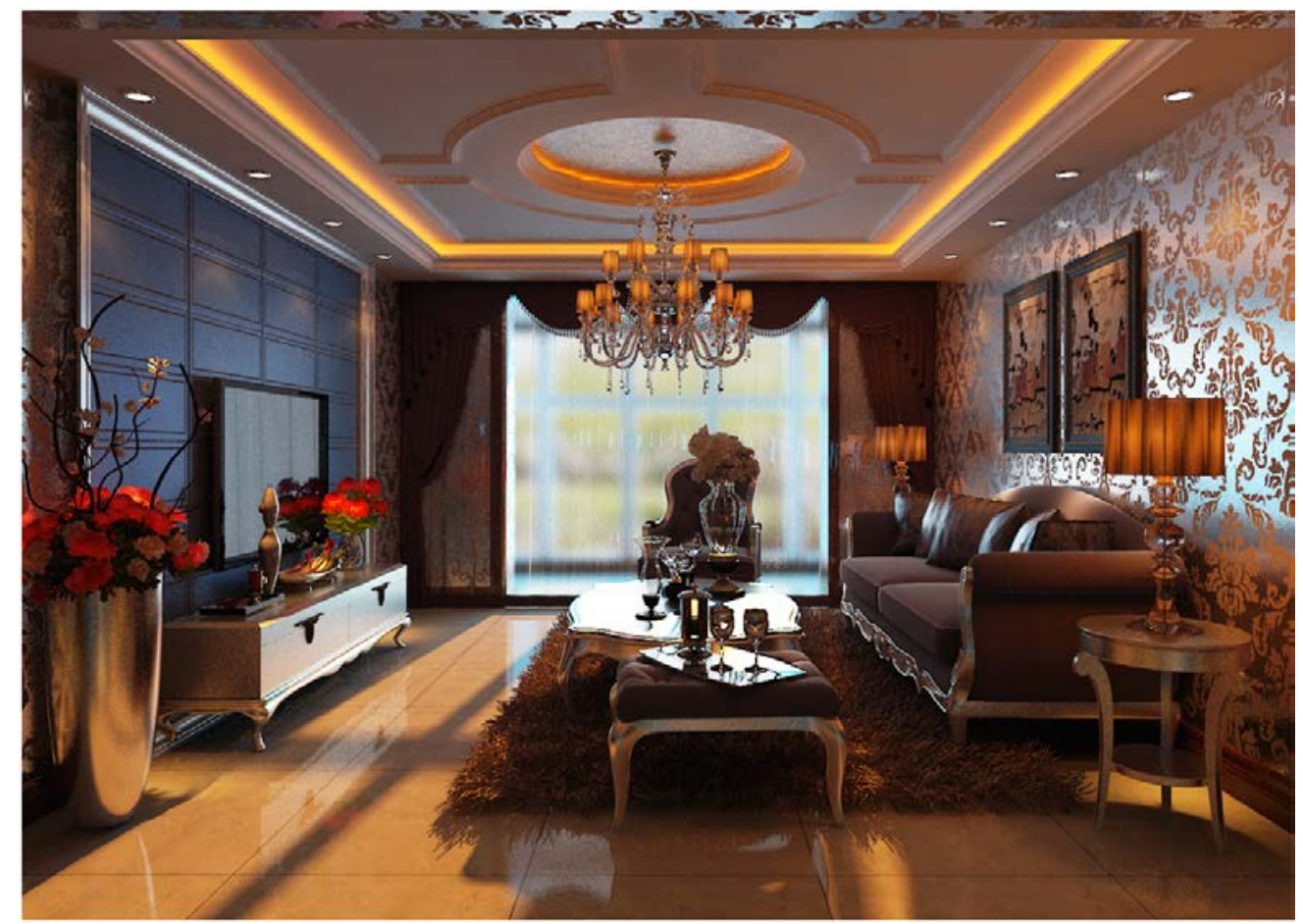

Fig 4. The overall effect of the living room

\section{Conclusion}

As the pace of life is continuing increasing, more and more people aspire to comfortable upscale living environment. The community use villa are generally high-end crowd, their design needs are more reflected in the spiritual and cultural level. The application of European style in villa design not only can create an elegant environment, but also highlight a certain status. European-style has a long history in interior design and has an absolute market share in modern villa design. It has an important theoretical and practical significance to have a better application of European style in villa design.

\section{References}

[1] H. Zhao, K.Y. Hou. The European-style application in modern villa space design, J. Kaneda, 2013, 08: 102.

[2] H.N. Zhang. The soft furnishings and decorative hardware in interior design, J. Urban Architecture, 2013, 12: $37+39$.

[3] Y.Y. Pan, The symbolic language in European-style interior design, J. Popular Literature, 2012,10: 87-88.

[4] H. Kang. Healthwise. Carrot and Attractively, D. Northwest National University, 2013. 